\title{
The association between low skeletal muscle mass and delirium: results from the nationwide multi-centre Italian Delirium Day 2017
}

 \\ A. Cherubini ${ }^{8} \cdot \mathrm{M}_{\mathrm{B}} \mathrm{Bo}^{9} \cdot \mathrm{E}_{\text {. Mossello }}{ }^{10}$ - A. Marengoni ${ }^{11}$. G. Bellelli ${ }^{12}$ on behalf of the Italo-Hispanic Study Group of \\ Delirium
}

Received: 10 June 2021 / Accepted: 31 July 2021 / Published online: 20 August 2021

(c) The Author(s) 2021

\begin{abstract}
Introduction Delirium and sarcopenia are common, although underdiagnosed, geriatric syndromes. Several pathological mechanisms can link delirium and low skeletal muscle mass, but few studies have investigated their association. We aimed to investigate (1) the association between delirium and low skeletal muscle mass and (2) the possible role of calf circumference mass in finding cases with delirium.

Methods The analyses were conducted employing the cross-sectional "Delirium Day" initiative, on patient 65 years and older admitted to acute hospital medical wards, emergency departments, rehabilitation wards, nursing homes and hospices in Italy in 2017. Delirium was diagnosed as a $4+$ score at the 4-AT scale. Low skeletal muscle mass was operationally defined as calf circumference $\leq 34 \mathrm{~cm}$ in males and $\leq 33 \mathrm{~cm}$ in females. Logistic regression models were used to investigate the association between low skeletal muscle mass and delirium. The discriminative ability of calf circumference was evaluated using non-parametric ROC analyses.

Results A sample of 1675 patients was analyzed. In total, $73.6 \%$ of participants had low skeletal muscle mass and $24.1 \%$ exhibited delirium. Low skeletal muscle mass and delirium showed an independent association (OR: 1.50; 95\% CI 1.09-2.08). In the subsample of patients without a diagnosis of dementia, the inclusion of calf circumference in a model based on age and sex significantly improved its discriminative accuracy [area under the curve (AUC) 0.69 vs $0.57, p<0.001$ ].

Discussion and conclusion Low muscle mass is independently associated with delirium. In patients without a previous diagnosis of dementia, calf circumference may help to better identify those who develop delirium.
\end{abstract}

Keywords Delirium $\cdot$ Older persons $\cdot$ Sarcopenia

\section{Introduction}

Delirium is a common and severe neuropsychiatric condition, characterized by an acute and fluctuating disorder of attention and cognitive function [1]. It is strongly associated with increased mortality, risk of re-hospitalization,

A. Zucchelli and F. Manzoni contributed equally.

A. Marengoni and G. Bellelli contributed equally.

Members of the "On Behalf of the Italo-HispanicStudy Group of Delirium" are listed in Acknowledgement section.

Alberto Zucchelli

a.zucchelli001@unibs.it

Extended author information available on the last page of the article institutionalization, higher costs of health services [1] and increased distress for patients, caregivers and health care providers [2,3].

Older persons are at greater risk of developing delirium: between 25 and $33 \%$ of older adults who are admitted to a medical ward develop this syndrome, either as the presenting sign of underlying acute illnesses or during the hospitalization [4-6].

The reduction of muscle mass is another common condition among older persons: each decade after age 30, between 3 and $8 \%$ of muscle mass is involuntarily lost [7]. Low skeletal muscle mass (SMM) is often associated with low muscle strength and poor functional performance, configuring the diagnosis of sarcopenia [8]. Similarly to those with delirium, persons with sarcopenia have increased risk of death, hospitalization, and of developing disability and loss of autonomy 
[8]. Sarcopenia is a main feature of frailty [9], which has been found associated with a higher risk of delirium [10].

However, few studies [11] so far have evaluated the overlap and the association between delirium and low SMM. These two conditions might have common pathogenetic mechanisms, such as inflammation, nutritional deficit, and low mobility $[12,13]$. Furthermore, they may influence each other when simultaneously present: for example, a reduction in muscle mass may alter the distribution volumes of drugs and thus their pharmacokinetic, enhancing the risk of druginduced delirium [14]. Lastly, both conditions, although common and impactful, are often underdiagnosed $[1,8]$ even if rapid screening and diagnostic tools are available.

In this study, we aimed to investigate if low SMM, defined as a reduced calf circumference, is independently associated with delirium. Further, we aimed to investigate the possible role of calf circumference in finding cases with delirium.

\section{Materials and methods}

\section{Study population}

We analyzed data from the Italian Delirium Day, 2017 edition. The aim and the details of the Delirium Day studies have been described elsewhere [5, 6]. Briefly, the physicians belonging to 12 Italian scientific societies were invited to participate in the study. Data about patients older than 65 years and admitted to the participating centers were collected from 00:00 to 23:59 on the 27th of September 2017. Demographics and information about medical history and pharmacological treatment were retrieved through medical records review, interview, and physical examination. Length of stay was calculated as the number of days between admission and the index day. Patients affected by aphasia, blindness, deafness, in a coma or in terminal conditions were excluded. An informed consent form was signed by either the patient or, in case of delirium or severe cognitive decline, by a proxy. Detailed information about data collection methodologies was provided and all data were gathered by the participating physicians using a web-based form. The study was approved by the Brianza Ethics Committee (protocol no. 2572). This study complies with the Declaration of Helsinki ethical standards.

In total, 3751 patients were recruited for the 2017 Delirium Day edition. Those who did not met inclusion criteria $(n=341)$ were excluded. Further, 127 were excluded because they missed information about demographics or delirium and 1582 were excluded because of missing data on calf circumference. After excluding 26 patients because the recorded value of calf circumference was an outlier, a total sample of 1675 patients was analyzed (Figure S1).

\section{Delirium assessment}

Delirium was assessed employing the 4AT [15], a rapid and validated tool for delirium detection. In a recent metanalysis, it showed high sensitivity and specificity (0.86 and 0.89 , respectively) in the diagnosis of delirium against reference standards [16]. Shortly, the tool is based on 4 items investigating acute changes in alertness, attention, and cognition. The probability of delirium is stratified according to the final score of the 4AT as follows: absence of delirium ( 0 points), unlikely diagnosis (1-3 points) or probable diagnosis (4+ diagnosis). In our study, a 4AT score of $4+$ points was used to diagnose delirium.

\section{Low skeletal muscle mass assessment}

Calf circumference was measured employing a semi-rigid tape around the widest part of the calf, applying a minimum pressure in order not to compress the subcutaneous tissue.

Calf circumference was dichotomized to operationally define low SMM, using sex-specific cut-offs for its identification ( $\leq 34 \mathrm{~cm}$ for males, $\leq 33 \mathrm{~cm}$ for females) [17-19].

\section{Statistical analyses}

Calf circumference outliers were identified by dividing the study population in 8 age-groups and assessing the distribution of calf circumference in each group: if the calf circumference was higher than the 3rd quartile or lower than the 1 st quartile by more than three times the interquartile range, the value was considered an outlier and the participant was excluded.

Study population's characteristics were described using absolute number and proportion, mean and standard deviation (SD) or median and interquartile range (IQR), as 
appropriate. Differences between patients with a diagnosis of delirium and those without were investigated by means of chi-squared test, t test or Mann-Whitney $U$ test, as appropriate. The associations between calf circumference and delirium were evaluated employing logistic regression models, both unadjusted and adjusted for major confounders. To investigate the role of calf circumference in delirium detection, the discriminative ability of different logistic regression models has been assessed using the area under the curve (AUC) from non-parametric ROC analyses. The relative improvement in terms of goodness-of-fit between models was calculated as the difference between the AUC of the evaluated model and the one of the reference model, divided by the AUC of the reference model. The AUCs of the logistic regression models were compared using bootstrapping technique $(n=2000)$. Non-parametric ROC analyses were used to assess the AUC and sensitivity/specificity of calf circumference alone in the detection of delirium among participants without dementia, stratifying by sex.

All analyses were conducted with R 4.0.3 (R Foundation for Statistical Computing, Vienna, Austria [20]) with an alpha level $=0.05$ (Table 1$)$.

\section{Results}

The mean age of the 1675 participants was 83.1 and $36.9 \%$ were males. The study population was mostly recruited in medical wards $(47.1 \%)$, followed by nursing homes $(29.9 \%)$, rehabilitation units $(16.2 \%)$, surgical wards $(4.9 \%)$, and palliative care units $(1.9 \%)$. The median calf circumference was $31.0 \mathrm{~cm}(\mathrm{IQR}=6.0 \mathrm{~cm})$. The participants excluded from this study because of missing values of calf circumference were younger, more likely to be male, without a previous diagnosis of dementia, and to be admitted to a general surgery ward or an intensive care unit in comparison with those included (all $p<0.050$ ). The prevalence of delirium was similar between the two groups $(p=0.514)$.

Calf circumference was associated with delirium, even after adjustment for age, sex, dementia diagnosis, CCI, recruitment setting, and ischemic heart disease diagnosis (Table 2). In the adjusted model, each centimeter more in calf circumference was associated with a $5 \%$ decrease in the probability of exhibiting delirium. Participants with low SMM were 1.5 times more likely to have delirium (adjusted odds ratio 95\% confidence interval 1.50-1.09, 2.08).

A logistic regression model based on age, sex and dementia diagnosis showed a discriminative ability in identifying delirium cases of 0.69 (Table 3). Including in the model the Charlson's comorbidity index, the length of stay (shown to be associated with delirium in univariate analyses, Table S1) and the calf circumference improved the goodness-of-fit by $15.9 \%$ (AUC 0.80, $p<0.001$ ).

Among participants without a diagnosis of dementia, a logistic regression model based on age and sex showed a discriminative ability lower than 0.60 . Including the Charlson's comorbidity index and the length of stay lead to an AUC of 0.63. The further inclusion of the calf circumference improved the AUC of the model by more than $20 \%$, in comparison with the reference one (AUC 0.69, $p<0.001$ ).

Figure 1 shows the ROC curves of calf circumference alone (unadjusted) in identifying delirium cases among patients without a diagnosis of dementia, in males and females. The AUC was 0.68 for both genders. The cut-offs used for the diagnosis of low SMM exhibited a sensitivity of 0.83 for both genders. Specificity was lower among females $(0.28)$ than among males $(0.32)$.

\section{Discussion}

In this multi-center study, we show that sarcopenia, measured by means of calf circumference, is independently associated with delirium and that, in patients without a previous diagnosis of dementia, calf circumference may help to identify delirium cases.

In our study, the prevalence of delirium was similar to the one reported in the literature [20], confirming the association between delirium and age, dementia, and the presence of multiple chronic conditions as already described [21].

Conversely, the proportion of patients with low calf circumference was higher than the one previously reported (ranging between 7 and 24\% [19, 22-26]). In most of these studies, the mean calf circumference exhibited by those affected by low skeletal muscle mass (range 31.5-32.4 cm) was higher than the one we found. In one study [25], including black South African women, the mean calf circumference for those diagnosed with low SMM according to DXA $(28 \mathrm{~cm})$ was similar the one found in our sample. Most studies, however, enrolled participants living in the community. Indeed, this finding may be, at least partially, explained by the fact that our study population was on average older than 80 years old, admitted to a hospital ward or living in nursing homes, and with high prevalence of chronic conditions and dementia, given the association of all these factors with low muscle mass and sarcopenia [8, 27-29].

A previous study [11] reported an association between sarcopenia (defined according to EGWSOP criteria) and delirium in hospitalized patients in Italy, confirming our results. Several pathogenic mechanisms might explain the association we found between low SMM and delirium. Low muscle mass and sarcopenia are strongly linked with frailty, in particular with its physical phenotype $[9,30]$ : patients 
Table 1 Characteristics of the study population, stratified according to calf circumference

\begin{tabular}{lccc}
\hline & $\begin{array}{l}\text { Whole sample } \\
(n=1675)\end{array}$ & $\begin{array}{l}\text { Without low skeletal muscle } \\
\text { mass }(n=443)(26.4 \%)\end{array}$ & $\begin{array}{l}\text { With low skeletal muscle } \\
\text { mass }(n=1232)(73.6 \%)\end{array}$ \\
\hline Age, mean (SD) & $83.1(7.6)$ & $81.3(7.6)$ & $83.8(7.5)$ \\
Male gender (\%) & $618(36.9)$ & $177(40.0)$ & $441(35.8)$ \\
Setting (\%) & & & $594(48.2)$ \\
Medical ward & $789(47.1)$ & $195(44.0)$ & $371(30.1)$ \\
Nursing home & $501(29.9)$ & $130(29.3)$ & $186(15.1)$ \\
Rehabilitation unit & $272(16.2)$ & $86(19.4)$ & $55(4.5)$ \\
Surgical ward & $82(4.9)$ & $27(6.1)$ & $26(2.1)$ \\
Palliative care unit & $31(1.9)$ & $5(1.1)$ & $3.0(4.0)$ \\
Charlson's comorbidity index, median (IQR) & $3.0(3.0)$ & $2.0(3.0)$ & $7.0(4.0)$ \\
Number of prescribed drugs, median (IQR) & $7.0(4.0)$ & $7.0(4.0)$ & $216(17.5)$ \\
Ischemic heart disease (\%) & $276(16.5)$ & $60(13.5)$ & $306(24.8)$ \\
Heart failure (\%) & $412(24.6)$ & $106(23.9)$ & $219(17.8)$ \\
Severe chronic kidney disease (\%) & $292(17.4)$ & $73(16.5)$ & $440(37.4)$ \\
Dementia (\%) & $555(34.8)$ & $115(27.6)$ & $12(135.0)$ \\
Length of stay, days, median (IQR) & $12(142.2)$ & $12(216.5)$ & $29.0(4.0)$ \\
Calf circumference, cm, median (IQR) & $31.0(6.0)$ & $36.0(3.0)$ & $328(29.3)$ \\
Delirium (\%) & $403(24.1)$ & $75(16.9)$ & 0.075
\end{tabular}

$S D$ standard deviation, $I Q R$ interquartile range

with lower calf circumference might be affected by frailty and, as such, at higher risk of developing delirium [10]. Furthermore, inflammatory cytokines and markers have been shown in the serum of persons with delirium [31] and in those affected by sarcopenia and low muscle mass [32]: abnormal and dysregulated inflammatory response might serve as trigger to both conditions. Changes in body composition typical of sarcopenic patients have been associated with increased risk of adverse drug reactions [14]: in consideration of the strong association between polypharmacy and delirium [33], it is likely that patients with reduced muscle mass are at higher risk of developing delirium even when therapeutic drug dosage are used. Our study strengthens previous results about the association of low muscle mass and delirium, broadening these findings to calf circumference, a simple proxy proposed for the evaluation of muscle quantity in the diagnostic algorithm of sarcopenia.

Of notice, among participants without a known diagnosis of dementia (26.8\% of delirium cases), calf circumference significantly improved the AUC of a model based on age, sex, comorbidities, and length of staying. Further, calf circumference alone showed a moderate discriminative ability in identifying delirium cases among participants without dementia. It is likely that when an overt dementia diagnosis is present, the discriminative ability of calf circumference in identifying those with delirium is weakened because of the strong association between dementia and delirium, which may overshadow the association between low SMM and delirium. Conversely, it is possible that, among those without a known diagnosis of dementia, the discriminative ability of calf circumference for delirium identification is higher, not only because of the aforementioned mechanisms, but also because it may identify those with prodromal or undiagnosed dementia, as lower muscle mass, sarcopenia, and cognitive impairment are reciprocally associated [24].

The results of our study should be read in light of some limitations. First, the Delirium Day is a point-prevalence study, preventing the possibility to investigate the causal relationship between low SMM and delirium. Second, although a high correlation between calf circumference and SMM has been reported [19, 22, 34], participants with oedema, obesity, or other conditions characterized by
Table 2 Associations of calf circumference measures with delirium

\begin{tabular}{lll}
\hline & Unadjusted OR (95\%CI) & Adjusted OR (95\%CI) \\
\hline Calf circumference & $0.95(0.93-0.97)$ & $0.95(0.92-0.97)$ \\
Low skeletal muscle mass & $1.78(1.35-2.36)$ & $1.50(1.09-2.08)$ \\
\hline
\end{tabular}

Adjustment: age, sex, Charlson's comorbidity index, dementia, ischemic heart disease, and setting OR odds ratio, $95 \%$ CI $95 \%$ Confidence intervals 
Table 3 goodness-of-fit (AUC) of different in logistic regression models in predicting delirium, in the whole sample and in the subsample of participants without a diagnosis of dementia

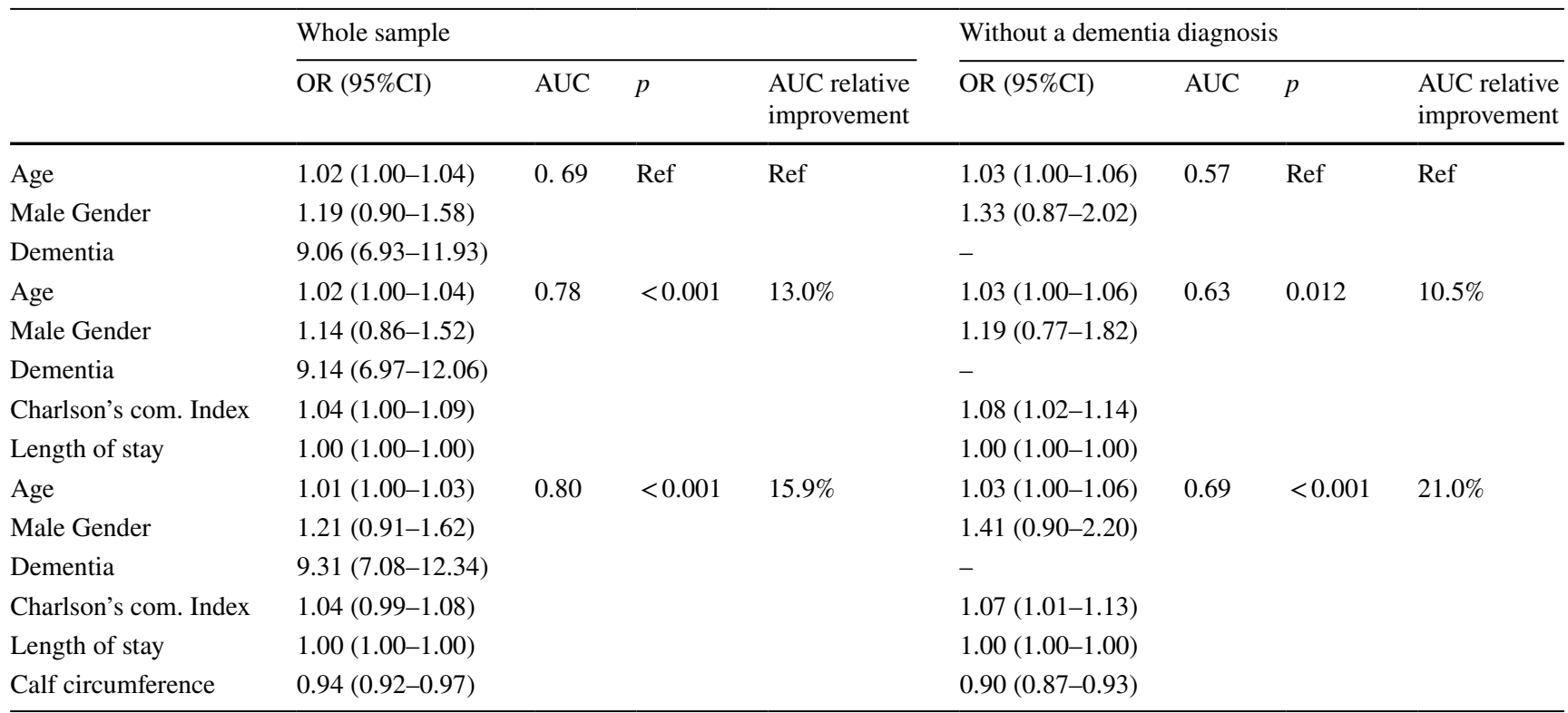

OR odds ratio, $95 \%$ CI 95\% Confidence Interval, $A U C$ area under the curve

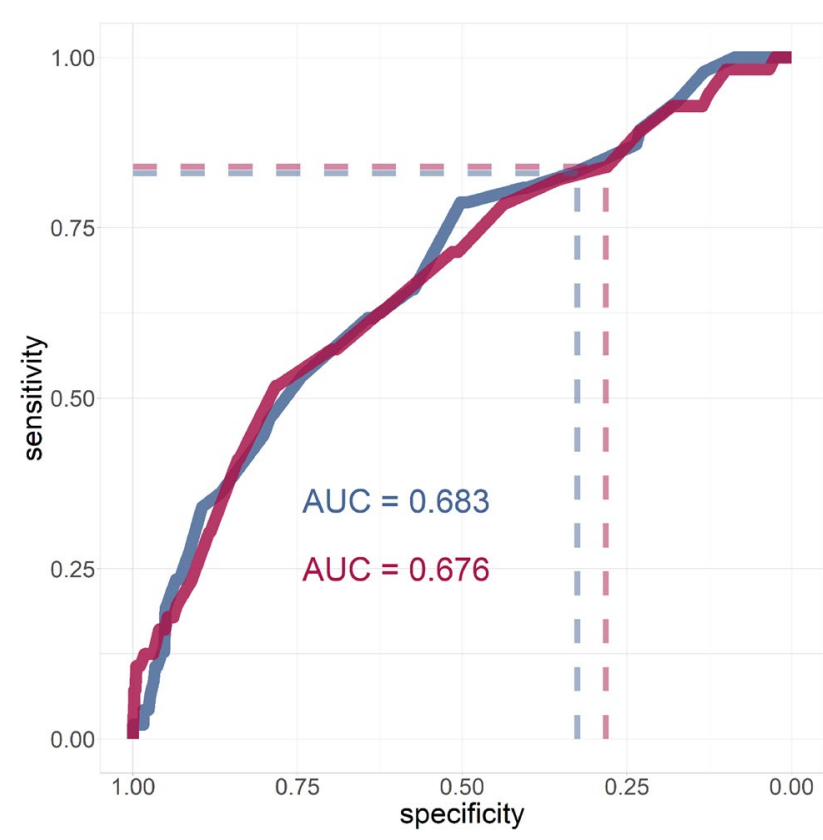

Fig. 1 Receiver-Operating-Characteristic curve for the identification of delirium in the subsample of patients without adiagnosis of dementia, stratified by sex (blue $=$ males, purple $=$ females)

increased calf circumference and decreased muscle mass could not be correctly identified, thus possibly hampering the reliability of such measure in a minority of patient [34, 35].

Some study strengths may also be considered. This is the first multi-center study identifying such association in a large cohort of older patients from several settings of care. Moreover, the diagnosis of delirium was obtained with a well-validated screening tool, which does not require preliminary training to be administered.

Our study has implications for the everyday clinical practice. Calf circumference is simple to measure by all healthcare workers in hospital and long-term care wards. For example, the calf circumference cut-offs suggested for the identification of low SMM exhibited high sensitivity in the identification of delirium cases. If future studies will confirm the study findings, calf circumference could be proposed as a method to identify patients at risk of developing delirium and other adverse outcomes.

\section{Conclusion}

Calf circumference is a rapid, easy to perform, and economic tool for SMM quantity evaluation: the inclusion of such simple measure in clinical practice might help to find cases of delirium, in particular those without manifest risk factors for delirium, such as dementia. 
Supplementary Information The online version contains supplementary material available at https://doi.org/10.1007/s40520-021-01950-8.

Acknowledgements On Behalf of the Italo-HispanicStudy Group of Delirium:

Investigators:Tarasconi A, Unità Operativa Chirurgia d'Urgenza, Azienda Ospedaliero-Universitaria Di Parma; Sella M, Auriemma S, Paternò G, Aulss 8 Berica, Vicenza; Faggian G, Lucarelli C, Azienda Ospedaliero-Universitaria di Verona; De Grazia N, Alberto C, Chirurgia AO Pugliese Ciaccio Di Catanzaro; Margola A, $1^{\wedge}$ Chirurgia Asst Spedali Civili di Brescia; Porcella L, Nardiello I, $3^{\wedge}$ Chirurgia Asst Spedali Civili di Brescia; Chimenti E, Zeni M, $2^{\wedge}$ Chirurgia Spedali Civili di Brescia; Giani A, Famularo S, Ospedale San Gerardo; Romairone E, Minaglia C, IRCCS Ospedale Policlinico San Martino, Genova; Ceccotti C, Guerra G, Mantovani G, Monacelli F, Minaglia C, Clinica Geriatrica Ospedale Policlinico San Martino, Genova; Candiani T, Medicina Generale - Geriatria; Ballestrero A, Minaglia C, Ospedale Policlinico San Martino, Genova; Santolini F, Minaglia C, Ospedale Policlinico San Martino, Genova; Rosso M, Bono V, Neurologia Ospedale Ss Annunziata; Sibilla S, Azienda Ospedaliera Pia Fondazione "Card. G. Panico"; Dal Santo P, Ceci M, Unità Operativa Complessa Geriatria Ulss 5 "Polesana"; Barone P, Schirinzi T, Azienda Ospedaliera Pia Fondazione "Card. G.Panico"; Formenti A, Nastasi G, Azienda Ospedaliera San Gerardo, Monza; Isaia G, Geriatria Azienda Ospedaliero-Universitaria San Luigi Gonzaga di Orbassano; Gonella D, Ospedale Galliera, Genova; Battuello A, Casson S, Ospedale di Chioggia Ulss 3 Serenissima, Mestre; Calvani D, Boni F, Soc Geriatria Nuovo Ospedale Santo Stefano - Prato- Azienda Asl Toscana Centro; Ciaccio A, Rosa R, Gastroenterologia; Sanna G, Manfredini S, Ospedale Civile Ss. Annunziata; Cortese L, Campus Bio-Medico di Roma; Rizzo M, Prestano R, Vi Medicina Interna Università della Campania Luigi Vanvitelli; Greco A, Lauriola M, S.C. di Geriatria; Gelosa G, Piras V, Grande Ospedale Metropolitano Niguarda; Arena M, Cosenza D, UOSD Stroke Unit; Bellomo A, LaMontagna M, S.P.D.C. Foggia, Asl Foggia, Ospedale Riuniti Foggia; Gabbani L, Lambertucci L, Sod Geriatria per La Complessità Assistenziale Azienda Ospedaliero-Universitaria Careggi; Perego S, Parati G, Istituto Auxologico Italiano Ospedale San Luca; Basile G, Uosd Geriatria; Gallina V, Pilone G, Azienda Ospedaliera Villa Scassi- As13; Giudice C, De F, Geriatria; Pietrogrande L, De B, Asst Santi Paolo E Carlo, Presidio S. Paolo; Mosca M, Corazzin I, Uo Complessa di Medicina Ospedale di Agordo; Rossi P, Nunziata V, Fondazione Irccs Ca' Granda Ospedale Maggiore Policlinico; D'Amico F, Grippa A, Unità Operativa Complessa Geriatria Po "Barone Ignazio Romeo " Patti; Giardini S, Reparto Geriatria per Acuti Ospedale Santa Maria Annunziata Usl Toscana Centro; Barucci R, Ortogeriatria Ospedale Santa Maria Annunziata Usl Toscana Centro; Cossu A, Fiorin L, Unità Operativa Medicina, Ospedale G.P. Delogu; Arena M, Distefano M, Uosd Neurofisiopatologia E Disordini del Movimento; Lunardelli M, Brunori M, Uo Geriatria; Ruffini I, Abraham E, Reparto di Geriatria Merano; Varutti A, Fabbro E, Degenze Chirurgiche; Catalano A, Martino G, Policlinico Universitario "G. Martino" di Messina; Leotta D, Marchet A, Struttura Complessa Neurologia; Dell'Aquila G, Scrimieri A, Inrca; Davoli M, Casella M, Arcispedale Santa Maria Nuova -Irccs - Soc Geriatria; Cartei A, Polidori G, Medicina Interna E Post-Chirurgica Azienda Ospedaliero Universitaria Careggi; Basile G, Brischetto D, Unità Operativa Complessa Medicina Delle Malattie Metaboliche; Motta S, U.O Cardiologia - Asst Monza; Saponara R, Asst Crema, Ospedale Maggiore; Perrone P, Asst Ovest Milanese; Russo G, A.O.U Federico Ii Di Napoli; Del D, Car C, Unità Operativa Complessa Neurologia, Ospedale Versilia, Azienda Usl Toscana Nord Ovest; Pirina T Unità Operativa di Geriatria; Franzoni S, Dipartimento Ortopedia E Traumatologia Fondazione Poliambulanza Istituto Ospedaliero; Cotroneo A, Ghiggia F, Reparto Geriatria Obdv; Volpi G, Menichetti C, Ospedale San Iacopo Pistoia; Bo M, Panico A, A.O.U. Città della
Salute e della Scienza, Torino; Calogero P, Corvalli G, Geriatria Calogero Policlinico Sant'Orsola Malpighi; Mauri M, Ospedale Circolo e Fondazione Macchi Varese; Lupia E, Medicina D'Urgenza - Mecau A.O.U. Città della Salute e della Scienza, Torino; Manfredini R, Fabbian F, Clinica Medica; March A, Pedrotti M, Geriatria Ospedale Di Bolzano; Veronesi M, Strocchi E, UO Medicina Interna; Bianchetti A, Crucitti A, Istituto Clinico S. Anna, Brescia; Di Francesco V, Fontana G, Geriatria A Azienda Ospedaliera Universitaria Integrata Verona; Bonanni L, Barbone F, Clinica Neurologica Università di Chieti; Serrati C, UO Neurologia Ospedaliera con Centro Ictus, Savona; Ballardini G, Simoncelli M, Medicina Rimini; Ceschia G, Scarpa C, Struttura Complessa Geriatria; Brugiolo R, Fusco S, Unità Operativa Complessa Geriatria - Ospedale Dell'Angelo, Trieste; Ciarambino T, Medicina Interna Marcianise; Biagini C, Tonon E, Unità Operativa Complessa Geriatria; Porta M, Sc Medicina 1U - Molinette Torino; Venuti D, Ospedale Evangelico Internazionale Genova; DelSette M, Poeta M, Ente Ospedaliero Ospedali Galliera, Genova; Barbagallo G, Trovato G, Medicina Interna P.O. Basilotta Nicosia (En) Asp 4, Enna; Delitala A, Clinica Medica - Azienda Ospedaliero-Universitaria di Sassari; Arosio P, Reggiani F, Humanitas Research Hospital, Rozzano, Milano; Zuliani G, Ortolani B, Arcispedale Sant'Anna di Cona; Mussio E, Fondazione Poliambulanza Istituto Ospedaliero, Brescia; Girardi A, Coin A, Clinica Geriatrica, Padova; Ruotolo G, Castagna A, Geriatria A.O. Pugliese Ciaccio di Catanzaro; Masina $\mathrm{M}$, Unità Operativa Complessa Geriatria Bentivoglio; Cimino R, Soc Medicina Interna; Pinciaroli A, Soc Nefrologia Ao Pugliese Ciaccio di Catanzaro; Tripodi G, Soc Ortopedia; Cannistrà U, Soc Neurologia; Cassadonte F, Vatrano M, Utic Ao Pugliese Ciaccio di Catanzaro; Cassandonte F, Soc Cardiologia; Scaglione L, Medicina Interna 5; Fogliacco P, Muzzuilini C, Medicina Generale Presidio Ospedaliero Di Ceva Aslcn1; Romano F, S.C. Medicina Interna; Padovani A, Rozzini L, UO Neurologia Asst Spedali Civili di Brescia; Cagnin A, Fragiacomo F, Clinica Neurologica, Dipartimento di Neuroscienze, Università Di Padova; Desideri G, Liberatore E, Ospedale Ss. Filippo E Nicola; Bruni A, Orsitto G, UOC. Medicina Interna P.O. "di Venere", L'Aquila; Franco M, Bonfrate L, Unità Operativa Complessa Geriatria Ospedale Generale Regionale F. Miulli; Bonetto M, S.C. Geriatria; Pizio N, Uo Neurologia; Magnani G, Cecchetti G, Ospedale San Raffale, Milano; Longo A, Bubba V, S.C. Geriatria; Marinan L, Ospedale San Bassiano; Cotelli M, Turla M, Asst Valcamonica; Brunori M, Medicina Interna Zoli; Sessa M, Abruzzi L, UO Neurologia Asst Cremona Ospedale Di Cremona; Castoldi G, LoVetere D, Unità Operativa Complessa Ortopedia e Traumatologia, Vimercate; Musacchio C, Ortogeriatria Eo Galliera; Novello M, Cavarape A, Clinica Medica; Bini A, Leonardi A, Asl Roma 1, Presidio Ospedaliero S. Filippo Neri Unità Operativa Complessa Medicina Interna, Roma; Seneci F, $1^{\circ}$ Traumatologia-Ortopedia Asst Spedali Civili di Brescia; Grimaldi W, Unità Operativa Geriatria Ospedale di Magenta (Mi) Asst Ovest Milanese; Seneci F, $2^{\circ}$ Traumatologia-Ortopedia Asst Spedali Civili di Brescia; Fimognari F, Bambara V, UOC. Geriatria Azienda Ospedaliera di Cosenza; Saitta A, Corica F, Medicina Interna; Braga M, Neurologia Asst Vimercate; Servi Medicina Interna Universitaria; Ettorre E, Camellini C, Policlinico Umberto I; Marengoni A, Spedali Civili Presidio Montichiari, Brescia; Bruni A, Crescenzo A, Ospedale Giovanni Paolo Ii Asp Cz; Noro G, Turco R, Ospedale Santa Chiara, Trento; Ponzetto M, Giuseppe L, Ospedale Civico di Chivasso Asl To4, Torino; Mazzei B, Maiuri G, Irccs_Inrca Ospedale Cosenza Unità Operativa Complessa di Geriatria; Costaggiu D, Medicina Generale; Damato R, Fabbro E, Ass3 "Alto Friuli-Collinare-Mediofriuli" - Presidio Ospedaliero Sd-T Degenze Internistiche Sd; Formilan M, Az. Ulss 3 Geriatria Dolo; Patrizia G, Santuari L, Fondazione Sanità e Ricerca; Gallucci M, Minaglia C, Ospedale Policlinico San Martino, Genova; Paragona M, Fondazione Hospice M.T.C. Seràgnoli Onlus; Bini P, Fondazione Terasa Camplani Cdc Ancelle; Modica D, Hospice Kairòs Siracusa; Abati C, Clerici M, Casa Hospice Cima Verde; Barbera I, Hospice Via Delle Stelle; NigroImperiale F, Hospice San Giuseppe Moscati; Manni A, Votino C, 
"Hospice Casa Madonna Dell'Uliveto" Scsrl; Castiglioni C, Di M, Asst Ovest Milanese; Degl'Innocenti M, Moscatelli G, Hospice Di Abbiategrasso; Guerini S, Fondazione Ips Card. Gusmini Onlus; Casini C, Hospice Convento Delle Oblate- Ufc Cure Palliative-Usl Toscana Centro-Firenze; Dini D, Unità Operativa di Terapie Palliative/Hospice; D'Imporzano E, Hospice Livorno; DeNotariis S, Pronto Soccorso E Medicina D'Urgenza, Policlinico Sant'Orsola Malpighi; Bonometti F, Pronto Soccorso Gardone Val Trompia, Brescia; Paolillo C, Riccardi A, Azienda Sanitaria Universitaria Integrata di Udine; Tiozzo A, SamySalamaFahmy A, Ospedale di Chioggia Ulss 3 Serenissima; Riccardi A, Paolillo C, Reparto di Medicina D'Urgenza; DiBari M, Vanni S, Pronto Soccorso Ospedale Careggi, Firenze; Scarpa A, Zara D, Ospedale Villa Salus; Ranieri P, Istituto Clinico S. Anna; Calogero P, Corvalli G, Pare (Post Acuti Riabilitazione Estensiva) Policlinico Sant'Orsola Malpighi; Pezzoni D, Fondazione Nobile Paolo Richiedei, Gussago Brescia; Gentile S, Morandi A, Platto C, D'Ambrosio V, Faraci B, Brambilla C. Fondazione Teresa Camplani - Casa di Cura Ancelle della Carità - Cremona; Ivaldi C, Cure Intermedie Ospedale Gallino; Milia P, DeSalvo F, Istitut Prosperius Tiberino; Solaro C, Strazzacappa M, Casa Di Cura "Mons. Luigi Novarese"; Bo M, Panico A, Istituto Di Riposo per La Vecchiaia I.R.V; Cazzadori M, Confente S, Lungodegenza San Bonifacio, Verona; Bonetto M, S.S. Cure Intermedie; "Grasso M, Troisi E, IRCCS Fondazione Santa Lucia; " Magnani G, Cecchetti G, Ospedale San Raffaele, Milano; Guerini V, Ips Fondazione Cardinal Gusmini di Vertova; Bernardini B, Corsini C, Neuroriabilitazione Humanitas, Rozzano, Milano; Boffelli S, Fondazione Poliambulanza, Brescia; Filippi A, Delpin K, Lungodegenza Post Acuzie; Bertoletti E, Ospedale Santa Viola; Vannucci M, Tesi F, Casa Di Cura Villa Ulivella E Glicini; Crippa P, Malighetti A, Fondazione Camplani Domus Salutis, Brescia; "Caltagirone C, DiSant S, IRCCS Fondazione Santa Lucia, Roma; " Bettini D, Maltese F, Spedali Civili Presidio Montichiari - Subacuti, Brescia; Formilan M, Az. Ulss 3 Lungodegenza Geriatrica Dolo; Abruzzese G, Minaglia C, Irccs Ospedale Policlinico San Martino; Cosimo D, Azienda Ospedaliera Pia Fondazione "Cardinale G.Panico"; Azzini M, Cazzadori M, Azienda Ulss 9 Scaligera, Verona; Colombo M, Procino G, Istituto Geriatrico "Camillo Golgi"; Fascendini S, Barocco F, Centro di Eccellenza Alzheimer Ferb Onlus - Ospedale Di Gazzaniga (Bergamo); Del P, Azienda Ospedaliera Pia Fondazione "Card. G.Panico"; D'Amico F, Grippa A, Uo Lungodegenza Po "Barone Ignazio Romeo" Patti; Mazzone A, Riva E, Istituto Piero Redaelli, Milano; Dell'Acqua D, Cottino M, Istituto Golgi Abbiategrasso, Pavia; Vezzadini G, Avanzi S, Ics Maugeri Castelgoffredo; Orini S, IRCCS Fatebenefratelli S. Giovanni Di Dio, Brescia; Sgrilli F, Mello A, Presidio P.Thouar; Lombardi L, Fondazione Istituto Ospedaliero di Sospiro Onlus, Cremona; Muti E, Fondazione Mons. A. Mazzali Onlus Mantova; Dijk B, Rsa Chiavari; Fenu S, Pes C, Policlinico Sassarese; Gareri P, Castagna A, CDCD Catanzaro Lido; Passamonte M, De F, Cure Sub Acute; Rigo R, Locusta L, Villaggio Amico, Gerenzano Varese; Caser L, Rosso G, Casa Della Divina Provvidenza Cottolengo di Biella; Cesarini S, Fontenuovo - Residenze Di Ospitalità Per Anziani - Fondazione Onlus; Cozzi R, Santini C, Rsa Virgilio Ferrari; Carbone P, Cazzaniga I, Rsa Oasi Domenica; Lovati R, Cantoni A, Rsa "Casa Per Coniugi"; Ranzani P, Rsa G.Gemellaro Onlus; Barra D, Pompilio G, Apsp Opera Romani; Dimori S, Fondazione Angelo Poretti E Angelo Magnani; Cernesi S, Riccò C, Cra Casa Serena; Piazzolla F, Capittini E, Rsa Parco Del Welfare; Rota C, Gottardi F, I.P.S. Fondazione Cardinal Gusmini; Merla L, Fondazione Ips Cardinal Gusmini Onlus, Vertova, Bergamo; Barelli A, Rsa Monticello; Millul A, Istituti Riuniti Airoldi E Muzzi; De G, Morrone G, C.R.A. "E. Cialdini"; Bigolari M, Minaglia C, Ospedale Policlinico San Martino; Macchi M, Zambon F, Piccola Casa Della Divina Provvidenza (Cottolengo); D'Amico F, Rsa Sant'Angelo Di Brolo; D'Amico F, Rsa "San Giovanni Di Dio" - Patti; Pizzorni C, Rsa Celesia Mantenimento; DiCasaleto G, Residenza San Gottardo; Menculini G, Marcacci M, Residenza Protetta "Creusa Brizi Bittoni"; Catanese G, Sprini D, Rsa Buon Pastore; DiCasalet T, Casa Di Riposo San Gottardo, Genova;
Bocci M, Centro Di Rianimazione; Borga S, Caironi P, Scdu Anestesia E Rianimazione; Cat C, Cingolani E, Rianimazione 1; Avalli L, Greco G, Terapia Intensiva Cardio-Toraco-Vascolare; Citerio G, Gandini L, Asst Monza Ospedale San Gerardo, Monza; Cornara G, Lerda R, Anestesia E Rianimazione; Brazzi L, Azienda Ospedaliero-Universitaria Città della Salute e della Scienza di Torino; Simeone F, Caciorgna M, Terapia Intensiva Cardiotoracica Azienda Ospedaliera Senese; Alampi D, Azienda Ospedaliera Sant'Andrea; Francesconi S, Beck E, ASST Monza - Ospedale di Desio - Terapia Intensiva Generale; Antonini B, Vettoretto K, Ospedale Di Manerbio ASST del Garda; Meggiolaro M, Azienda Ospedaliera Universitaria Padova - Rianimazione Centrale; Garofalo E, Bruni A, Azienda Ospedaliero-Universitaria Mater Domini; Notaro S, Terapia Intensiva Cardio Respiratoria-TicreOspedale Monaldi; Varutti R, Bassi F, Aas5 Friuli Occidentale; Mistraletti G, Marino A, Ospedale San Paolo; Rona R, Rondelli E, Rianimazione Generale Ospedale San Gerardo, Monza; Riva I, Anestesia 3 Ti Adulti; Scapigliati A, Policlinico Gemelli; Cortegiani A, Vitale F, Policlinico Paolo Giaccone. Università degli Studi di Palermo; Pistidda L, Cliniche Universitarie Azienda Ospedaliero-Universitaria - Sassari; D'Andrea R, Querci L, Policlinico Universitario Sant'Orsola-Malpighi; Gnesin P, Todeschini M, Terapia Intensiva - ASST Franciacorta, Brescia; Lugano M, Clinica Di Anestesia E Rianimazione Azienda Sanitaria Universitaria Integrata Udine; Castelli G, Ortolani M, Asst Mantova Ospedale Carlo Poma, Mantova; Cotoia A, Azienda Universitario Ospedaliera O.O.R.R.; Maggiore S, DiTizio L, Ospedale Ss. Annunziata; Graziani R, Testa I, unità Operativa Complessa Anestesia Rianimazione Ospedale Profili Di Fabriano (Ancona)-Asur Marche Av2; Ferretti E, Castioni C, Ospedale San Giovanni Bosco; Lombardi F, Caserta R, Fondazione Poliambulanza, Brescia; Pasqua M, Simoncini S, Asst Vallecamonica; Baccarini F, Unità Operativa Complessa Anestesia e Rianimazione - Ravenna; Rispoli M, Azienda Ospedaliera Dei Colli - Ospedale Monaldi, Napoli; "Grossi F, Cancelliere L, Centro di Rianimazione Scdu Anestesia E Rianimazione Azienda OspedalieroUniversitaria Maggiore Della Carità - Novara; " Carnelli M, Asst Lariana; Puccini F, Biancofiore G, Anestesia E Rianimazione TrapiantiAzienda Ospedaliero Universitaria Pisana; Siniscalchi A, Laici C, Uo Anestesia e Terapia Intensiva Polivalente e Dei Trapianti - Faenza; Mossello E, Torrini M, Geriatria-UTIC Careggi, Firenze; Pasetti G, San Giovanni Di Dio; Palmese S, Azienda Ospedaliera Universitaria San Giovanni di Dio e Ruggi D'Aragona; Oggioni R, Mangani V, Terapia Intensiva Ospedale S. Giovanni di Dio Firenze; Pini S, Martelli M, Azienda Ospedaliera Universitaria Pisana. Rianimazione Pisa; Rigo E, Terapia Intensiva Neurochirugica Treviso; Zuccalà F, Cherri A, $1^{\mathrm{a}}$ Unità di Terapia Intensiva Asst-Spedali Civili Di Brescia; Spina R, Calamai I, Anestesia E Rianimazione Presidio di Empoli; Petrucci N, Caicedo A, Terapia Intensiva, Asst-Garda, Brescia; Ferri F, Gritti P, Terapia Intensiva Neurochirurgica - Asst Papa Giovanni XXIII, Bergamo; Brienza N, Terapia Intensiva "De Blasi" Azienda Ospedaliera Universitaria Policlinico Bari; Fonnesu R, Dessena M, Ospedale Giovanni Paolo II; Fullin G, Saggioro D, Ospedale Dell'Angelo - Mestre.

Authors' contributions Conception and design of the work: AZ, FM, AM, GB. Data analysis: AZ. Interpretation of the results: all coauthors. Drafting the article: AZ, FM, AM, GB. Critical revision of the manuscript: all coauthors. Final approval of the manuscript: all coauthors. All the authors fulfill the ICMJE criteria for authorship.

Funding Open access funding provided by Università degli Studi di Brescia within the CRUI-CARE Agreement. This research did not receive any specific grant from funding agencies in the public, commercial, or not-for-profit sectors. 


\section{Declarations}

Conflict of interest All authors declare that no organizations have attempted to influence the study or the writing of the manuscript. They have had no financial relationships in the previous three years with any organizations that might have an interest in influencing the submitted work and no other relationships or activities that could appear to have influenced the submitted work.

Ethical standards compliance This study was carried out in keeping with the principles of the Declaration of Helsinki.

Ethical approval and informed consent The study was approved by the Brianza Ethics Committee (protocol n. 2572). An informed consent form was signed by either the patient or, in case of delirium or severe cognitive decline, by a proxy.

Human and animal rights statement All procedures performed in this study involving human participants were in accordance with the1964 Helsinkideclaration and its later amendments.

Transparency statement The lead authors (AZ and FM) affirm that the manuscript is an honest, accurate, and transparent account of the study being reported; that no important aspects of the study have been omitted; and that any discrepancies from the study as originally planned have been explained.

Open Access This article is licensed under a Creative Commons Attribution 4.0 International License, which permits use, sharing, adaptation, distribution and reproduction in any medium or format, as long as you give appropriate credit to the original author(s) and the source, provide a link to the Creative Commons licence, and indicate if changes were made. The images or other third party material in this article are included in the article's Creative Commons licence, unless indicated otherwise in a credit line to the material. If material is not included in the article's Creative Commons licence and your intended use is not permitted by statutory regulation or exceeds the permitted use, you will need to obtain permission directly from the copyright holder. To view a copy of this licence, visit http://creativecommons.org/licenses/by/4.0/.

\section{References}

1. Inouye SK, Westendorp RG, Saczynski JS (2014) Delirium in elderly people. Lancet 383:911-922. https://doi.org/10.1016/ S0140-6736(13)60688-1

2. Morandi A, Lucchi E, Turco R et al (2015) Delirium superimposed on dementia: a quantitative and qualitative evaluation of informal caregivers and health care staff experience. J Psychosom Res 79:272-280. https://doi.org/10.1016/j.jpsychores.2015.06.012

3. Morandi A, Lucchi E, Turco R et al (2015) Delirium superimposed on dementia: a quantitative and qualitative evaluation of patient experience. J Psychosom Res 79:281-287. https://doi.org/ 10.1016/j.jpsychores.2015.07.010

4. Marcantonio ER (2017) Delirium in hospitalized older adults. N Engl J Med 377:1456-1466. https://doi.org/10.1056/NEJMcp1605 501

5. Bellelli G, Morandi A, Di Santo SG et al (2016) "Delirium Day": a nationwide point prevalence study of delirium in older hospitalized patients using an easy standardized diagnostic tool. BMC Med 14:106. https://doi.org/10.1186/s12916-016-0649-8
6. Morandi A, Di Santo SG, Zambon A et al (2019) Delirium, Dementia, and In-Hospital Mortality: the results from the Italian Delirium Day 2016, A National Multicenter Study. J Gerontol Biol Sci Med Sci 74:910-916. https://doi.org/10.1093/gerona/ gly 154

7. Melton LJ 3rd, Khosla S, Crowson CS et al (2000) Epidemiology of sarcopenia. J Am Geriatr Soc 48:625-630

8. Cruz-Jentoft AJ, Bahat G, Bauer J et al (2019) Sarcopenia: revised European consensus on definition and diagnosis. Age Ageing 48:16-31. https://doi.org/10.1093/ageing/afy169

9. Fried LP, Tangen CM, Walston J et al (2001) Frailty in older adults: evidence for a phenotype. J Gerontol Biol Sci Med Sci 56:M146-M156. https://doi.org/10.1093/gerona/56.3.m146

10. Persico I, Cesari M, Morandi A et al (2018) Frailty and delirium in older adults: a systematic review and meta-analysis of the Literature. J Am Geriatr Soc 66:2022-2030. https://doi.org/10.1111/ jgs. 15503

11. Bellelli G, Zambon A, Volpato S et al (2018) The association between delirium and sarcopenia in older adult patients admitted to acute geriatrics units: results from the GLISTEN multicenter observational study. Clin Nutr 37:1498-1504. https://doi.org/10. 1016/j.clnu.2017.08.027

12. Martone AM, Bianchi L, Abete P et al (2017) The incidence of sarcopenia among hospitalized older patients: results from the Glisten study. J Cachexia Sarcopenia Muscle 8:907-914. https:// doi.org/10.1002/jcsm. 12224

13. Wilson D, Jackson T, Sapey E et al (2017) Frailty and sarcopenia: the potential role of an aged immune system. Ageing Res Rev 36:1-10. https://doi.org/10.1016/j.arr.2017.01.006

14. Hopkins JJ, Sawyer MB (2017) A review of body composition and pharmacokinetics in oncology. Expert Rev Clin Pharmacol 10:947-956. https://doi.org/10.1080/17512433.2017.1347503

15. Bellelli G, Morandi A, Davis DH et al (2014) Validation of the 4AT, a new instrument for rapid delirium screening: a study in 234 hospitalised older people. Age Ageing 43:496-502. https:// doi.org/10.1093/ageing/afu021

16. Tieges Z, Maclullich AMJ, Anand A et al (2020) Diagnostic accuracy of the 4AT for delirium detection in older adults: systematic review and meta-analysis. Age Ageing. https://doi.org/10.1093/ ageing/afaa224

17. Chen CY, Tseng WC, Yang YH et al (2020) Calf circumference as an optimal choice of four screening tools for sarcopenia among Ethnic Chinese older adults in assisted living. Clin Interv Aging 15:2415-2422. https://doi.org/10.2147/CIA.S287207

18. Barbosa-Silva TG, Bielemann RM, Gonzalez MC et al (2016) Prevalence of sarcopenia among community-dwelling elderly of a medium-sized South American city: results of the COMO VAI? study. J Cachexia Sarcopenia Muscle 7:136-143. https://doi.org/ 10.1002/jcsm. 12049

19. Kawakami R, Murakami H, Sanada K et al (2015) Calf circumference as a surrogate marker of muscle mass for diagnosing sarcopenia in Japanese men and women: Calf circumference and sarcopenia. Geriatr Gerontol Int 15:969-976. https://doi.org/10. 1111/ggi.12377

20. R Development Core Team (2010) R: A Language and Environment for Statistical Computing. R Foundation for Statistical Computing. http://www.polsci.wvu.edu/duval/PS603/Notes/R/ fullrefman.pdf. Accessed 22 Jul 2021

21. Geriatric Medicine Research Collaborative (2019) Delirium is prevalent in older hospital inpatients and associated with adverse outcomes: results of a prospective multi-centre study on World Delirium Awareness Day. BMC Med 17:229. https://doi.org/10. 1186/s12916-019-1458-7

22. Kawakami R, Miyachi M, Sawada SS et al (2020) Cut-offs for calf circumference as a screening tool for low muscle mass: 
WASEDA'S Health Study. Geriatr Gerontol Int 20:943-950. https://doi.org/10.1111/ggi.14025

23. Kim S, Kim M, Lee $\mathrm{Y}$ et al (2018) Calf circumference as a simple screening marker for diagnosing sarcopenia in older Korean Adults: the Korean Frailty and Aging Cohort Study (KFACS). J Korean Med Sci 33:e151. https://doi.org/10.3346/jkms.2018.33. e151

24. Tey SL, Chew STH, How CH et al (2019) Factors associated with muscle mass in community-dwelling older people in Singapore: findings from the SHIELD study. PLoS ONE 14:e0223222. https://doi.org/10.1371/journal.pone.0223222

25. Ukegbu P, Kruger H, Meyer J et al (2018) The association between calf circumference and appendicular skeletal muscle mass index of black urban women in Tlokwe City. J Endocrinol Metab Diabetes South Afr 23:86-90. https://doi.org/10.1080/16089677.2018. 1518825

26. Bahat G, Tufan F, Bahat $\mathrm{Z}$ et al (2013) Assessments of functional status, comorbidities, polypharmacy, nutritional status and sarcopenia in Turkish community-dwelling male elderly. Aging Male 16:67-72. https://doi.org/10.3109/13685538.2013.771329

27. Dodds RM, Granic A, Robinson SM et al (2020) Sarcopenia, longterm conditions, and multimorbidity: findings from UK Biobank participants. J Cachexia Sarcopenia Muscle 11:62-68. https://doi. org/10.1002/jcsm. 12503

28. Ogawa Y, Kaneko Y, Sato T et al (2018) Sarcopenia and muscle functions at various stages of Alzheimer Disease. Front Neurol 9:710. https://doi.org/10.3389/fneur.2018.00710

29. Chang KV, Hsu TH, Wu WT et al (2016) Association between sarcopenia and cognitive impairment: a systematic review and meta-analysis. J Am Med Dir Assoc 17:1164 e7-1164 e15. https:// doi.org/10.1016/j.jamda.2016.09.013

30. Bahat G, Ozkok S, Kilic C et al (2021) SARC-F Questionnaire detects frailty in older adults. J Nutr Health Aging 25:448-453. https://doi.org/10.1007/s12603-020-1543-9

31. Maldonado JR (2013) Neuropathogenesis of delirium: review of current etiologic theories and common pathways. Am J Geriatr Psychiatry 21:1190-1222. https://doi.org/10.1016/j.jagp.2013.09. 005

32. Bano G, Trevisan C, Carraro S et al (2017) Inflammation and sarcopenia: a systematic review and meta-analysis. Maturitas 96:10-15. https://doi.org/10.1016/j.maturitas.2016.11.006

33. Aloisi G, Marengoni A, Morandi A et al (2019) Drug prescription and delirium in older inpatients: results from the Nationwide Multicenter Italian Delirium Day 2015-2016. J Clin Psychiatry. https://doi.org/10.4088/JCP.18m12430

34. Gonzalez MC, Mehrnezhad A, Razaviarab N et al (2021) Calf circumference: cutoff values from the NHANES 1999-2006. Am J Clin Nutr 113:1679-1687. https://doi.org/10.1093/ajen/nqab029

35. Bahat G (2021) Measuring calf circumference: a practical tool to predict skeletal muscle mass via adjustment with BMI. Am J Clin Nutr 113:1398-1399. https://doi.org/10.1093/ajcn/nqab107

Publisher's Note Springer Nature remains neutral with regard to jurisdictional claims in published maps and institutional affiliations.

\section{Authors and Affiliations}

\section{Alberto Zucchelli ${ }^{1}$ (D) F. Manzoni ${ }^{2}$ - A. Morandi ${ }^{3,4}$ - S. Di Santo ${ }^{5,6} \cdot$ E. Rossi ${ }^{7} \cdot$ M. G. Valsecchi ${ }^{7}$ M. Inzitari ${ }^{4}$. A. Cherubini ${ }^{8} \cdot$ M. Bo $^{9} \cdot$ E. Mossello ${ }^{10} \cdot$ A. Marengoni ${ }^{11} \cdot$ G. Bellelli ${ }^{12}$ on behalf of the Italo-Hispanic Study Group of Delirium}

1 Department Information Engineering, Università Degli Studi Di Brescia, via Branze 38, 25123 Brescia, Italy

2 U.O. Medicina, ASST Spedali Civili, P.O. Montichiari, Montichiari, Italy

3 Department of Rehabilitation and Aged Care, "Fondazione Camplani" Hospital, Cremona, Italy

4 REFiT Bcn Research Group, Parc Sanitari Pere Virgili and Vall D'Hebron Institut de Recerca (VHIR), Barcelona, Spain

5 Department of Systems Medicine, Tor Vergata University, Rome, Italy

6 Department of Clinical and Behavioral Neurology, IRCCS Fondazione Santa Lucia, Rome, Italy

7 Bicocca Center of Bioinformatics, Biostatistics and Bioimaging, Università Degli Studi Di Milano-Bicocca, Monza, Italy
8 Geriatria, Accettazione Geriatrica e Centro Di Ricerca Per L'invecchiamento, POR, IRCCS INRCA, Ancona, Italy

9 Section of Geriatrics, Department of Medical Sciences, Università Degli Studi Di Torino, A.O.U. Città Della Salute E Della Scienza Di Torino, Turin, Italy

10 Department of Clinical and Experimental Medicine, Università Degli Studi Di Firenze, Firenze, Italy

11 Department of Clinical and Experimental Sciences, Università Degli Studi Di Brescia, Brescia, Italy

12 School of Medicine and Surgery, Università Degli Studi Milano-Bicocca and Acute Geriatric Unit, San Gerardo Hospital Monza, Milan, Italy 